www.nature.com/ejhg

\title{
Novel fibroblast growth factor receptor 3 (FGFR3) mutations in bladder cancer previously identified in non-lethal skeletal disorders
}

\author{
Bas WG van Rhijn ${ }^{1,2}$, Angela AG van Tilborg ${ }^{1}$, Irene Lurkin ${ }^{1}$, Jacky Bonaventure ${ }^{3}$, \\ Annie de Vries $^{1}$, Jean-Paul Thiery ${ }^{4}$, Theodorus $H$ van der Kwast ${ }^{1}$, Ellen C Zwarthoff ${ }^{*}{ }^{1}$ \\ and Francois Radvanyi ${ }^{4}$
}

\begin{abstract}
${ }^{1}$ Department of Pathology, Josephine Nefkens Institute, Erasmus University, 3000 DR Rotterdam, The Netherlands; ${ }^{2}$ Department of Urology, Erasmus University and University Hospital, 3000 CA Rotterdam, The Netherlands; ${ }^{3}$ Unité de Recherches sur les Handicaps Génétiques de l'Enfant, INSERM U 393, Hôpital Necker, 75743 Paris Cedex 15, France; ${ }^{4}$ Laboratoire de Morphogenèse Cellulaire et Progression Tumorale, UMR 144, CNRS, Institut Curie, 75248 Paris Cedex 05, France
\end{abstract}

Activating mutations in the fibroblast growth factor receptor 3 (FGFR3) gene are responsible for several autosomal dominant craniosynostosis syndromes and chondrodysplasias i.e. hypochondroplasia, achondroplasia, SADDAN and thanatophoric dysplasia - a neonatal lethal dwarfism syndrome. Recently, activating FGFR3 mutations have also been found to be present in cancer, i.e. at high frequency in carcinoma of the bladder and rarely in multiple myeloma and carcinoma of the cervix. Almost all reported mutations in carcinomas corresponded to the mutations identified in thanatophoric dysplasia. We here screened a series of 297 bladder tumours and found three FGFR3 somatic mutations (G380/ $382 \mathrm{R}$; $\mathrm{K} 650 / 652 \mathrm{M}$ and $\mathrm{K} 650 / 652 \mathrm{~T}$ ) that were not previously identified in carcinomas or thanatophoric dysplasia. Another novel finding was the occurrence of two simultaneous FGFR3 mutations in four tumours. Two of the three new mutations in bladder cancer, the G380/382R and the K650/652M mutations, were previously reported in achondroplasia and SADDAN, respectively. These syndromes entail a longer life span than thanatophoric dysplasia. The K650/652T mutation has not previously been detected in patients with skeletal disorders, but affects a codon that has been shown to be affected in some cases of thanatophoric dysplasia, SADDAN and hypochondroplasia. From a clinical perspective, the patients with FGFR3-related, non-lethal skeletal disorders might be at a higher risk for development of bladder tumours than the general population.

European Journal of Human Genetics (2002) 10, 819-824. doi:10.1038/sj.ejhg.5200883

Keywords: fibroblast growth factor receptor 3 (FGFR3); bladder; cancer; achondroplasia; hypochondroplasia; SADDAN; mutation

\section{Introduction}

The fibroblast growth factor receptor 3 (FGFR3) belongs to a family of structurally related tyrosine kinase receptors

\footnotetext{
*Correspondence: EC Zwarthoff; Department of Pathology, Josephine Nefkens Institute, Erasmus University, PO Box 1738, 3000 DR Rotterdam, The Netherlands; Tel: +31-104087929; Fax: +31-104089487;

E-mail: zwarthoff@path.fgg.eur.nl

Received 22 January 2002; revised 9 August 2002; accepted 13 August 2002
}

encoded by four different genes $(F G F R 1-4)$. These receptors consist of three glycosylated extracellular immunoglobulinlike domains (Ig-like), a transmembrane domain and a split intracellular tyrosine-kinase domain. Ligand binding induces FGFR dimerisation, resulting in autophosphorylation of the kinase domain and interaction with and phosphorylation of effector signalling proteins. ${ }^{1,2}$ Alternative mRNA splicing mechanisms generate many different receptor isoforms, which differ in ligand specificity. The 
isoforms $F G F R 3 b$ and $F G F R 3 c$ result from a mutually exclusive splicing event, in which the second half of the third Iglike domain is encoded by either the 151 nucleotides of exon 8 or the 145 nucleotides of exon $9 .^{3}$ These two isoforms have different tissue distributions: for example, FGFR $3 b$ is the main form in epithelial cells whereas FGFR3c is the predominant form in chondrocytes. ${ }^{3-5}$

Point mutations in specific domains of FGFR3 are associated with autosomal dominant dwarfism and craniosynostosis syndromes such as hypochondroplasia, achondroplasia (the most common form of skeletal dysplasia), severe achondroplasia with developmental delay and acanthosis nigricans (SADDAN), thanatophoric dysplasia (a lethal form of dwarfism), Crouzon syndrome with acanthosis nigricans and Muenke coronal craniosynostosis. ${ }^{3,6,7}$ Several reports have demonstrated that these mutations lead to constitutive activation of the receptor. ${ }^{8-11}$

In contrast with the inhibitory role on bone growth, an oncogenic role for FGFR3 in human cancer has emerged. Indeed, somatic activating mutations in FGFR3 have been reported in multiple myeloma and, more recently, in two epithelial malignancies, i.e. bladder- and cervix carcinomas. ${ }^{5,12,13}$ FGFR3 mutations are rare in multiple myeloma and cervix carcinomas, ${ }^{14,15}$ whereas their high incidence in bladder carcinomas $(74 \%$ of non-invasive papillary tumours) suggests that the constitutive activation of FGFR3 is an important event for bladder tumorigenesis. ${ }^{16,17}$ Nearly all mutations identified in bladder tumours are identical to the activating mutations responsible for thanatophoric dysplasia, a lethal form of dwarfism..$^{5,16-18}$ Only two of the 117 FGFR3 mutations identified by these groups, the A393E and K652Q mutations, do not correspond to thanatophoric dysplasia mutations. ${ }^{17,18}$ The A393E mutation is identical to a mutation associated with a craniosynostosis syndrome (Crouzon syndrome with acanthosis nigricans) and the K652Q mutation is identical to a mutation associated with hypochondroplasia. ${ }^{11,19}$

In this report, we describe three new somatic FGFR3 mutations in bladder tumours (G380/382R, K650/652M and K650/652T) (FGFR3c isoform numbering/FGFR3b isoform numbering). The G380/382R and the K650/652M mutations have previously been reported in achondroplasia and SADDAN, respectively. The K650/652T mutation has not been reported before in a skeletal disorder. In addition, among the 177 mutated tumours, we observed the occurrence of two concurrent FGFR3 mutations in four cases.

\section{Materials and methods}

FGFR3 mutation analysis was performed in a series of 297 bladder tumours as described. ${ }^{16}$ T7 Sequenase v2.0 (Amersham life Science, Inc., Cleveland, OH, USA) was used for sequencing to analyse the four regions of FGFR3, located in exons 7, 10, 15 and 19, known to harbour the point mutations previously described in multiple myeloma, bladder- and cervix carcinomas, thanatophoric dysplasia and
SADDAN. None of the 297 patients had a hereditary skeletal disorder documented. The mean patient's age at diagnosis was 65.9 years (range, 23-90). Seventy (24\%) of the 297 patients were female. The three new mutations of FGFR3 and all the 'double' mutations were confirmed on a second PCR product.

\section{Results}

In this series of 297 bladder carcinomas, we detected 181 FGFR3 mutations. Consistent with previous studies, ${ }^{5,16-18}$ most of the mutations (173 out of $181,96 \%$ ), were identical to the germinal activating mutations responsible for thanatophoric dysplasia, with the S249C mutation, found in 125 (69\%) of the 181 mutations as the most frequent point mutation. The A391/393E mutation, identical to the germinal mutation that causes Crouzon syndrome with acanthosis nigricans and already identified in bladder cancer, ${ }^{17}$ was found in four tumours.

We detected three mutations in this series of 297 bladder carcinomas, which were not previously identified in carcinomas or in thanatophoric dysplasia patients (Figure 1). These mutations affected the codons 380/382 and 650/652 (FGFR3c isoform numbering/FGFR3b isoform numbering). The G380/382R mutation affected the transmembrane domain and resulted in the replacement of a glycine by an arginine residue. This mutation is identical to a germinal activating mutation associated with the most common form of genetic dwarfism, i.e. achondroplasia. ${ }^{20-22}$ The other two mutations (K650/652M and K650/652T), affecting the tyrosine kinase domain, resulted in the replacement of a lysine by a methionine or a threonine. The $\mathrm{K} 650 / 652 \mathrm{M}$ mutation is identical to the germinal activating mutation that causes SADDAN. ${ }^{23}$ This novel mutation was found twice in this series of tumours. The matched constitutional DNA contained the wild-type sequence in every case, demonstrating the somatic nature of these mutations in (bladder) cancer. The tumour with the G380/382R mutation also displayed a S249C mutation in FGFR3. This patient suffered a recurrence 5 years later and the recurrent tumour was found to contain the same two FGFR3 mutations.

In addition to the above-mentioned case, three other bladder tumours also contained two distinct FGFR3 mutations. In each case, the S249C mutation was present. S249C was accompanied by the R248C, G370/372C and A391/393E mutations. The clinico-pathological data of the bladder-carcinoma patients carrying the new and the 'double' mutations are depicted together with the type of FGFR3 mutations in Table 1. We found no significant differences in the pathological status or the clinical data for the various FGFR3 mutations in bladder cancer (not shown).

Figure 2 indicates the locations and the nature of the missense mutations associated with skeletal disorders and bladder carcinomas. The frequencies of the various FGFR3 mutations in bladder cancer are also indicated. These 
A. $\quad G 380 / 382 R$

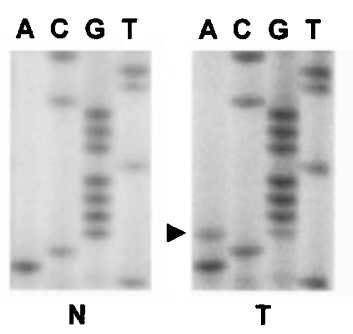

K650/652M

G A T C

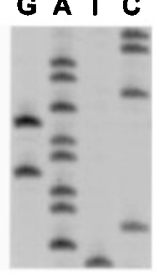

$\mathbf{N}$
G A T C

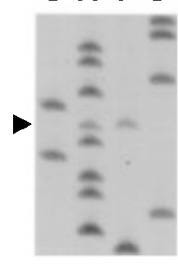

$\mathbf{T}$
K650/652T

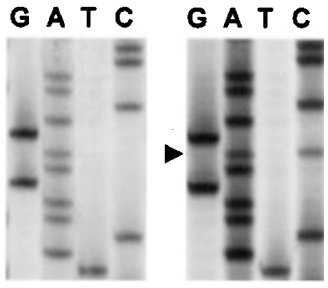

N

$\mathbf{T}$

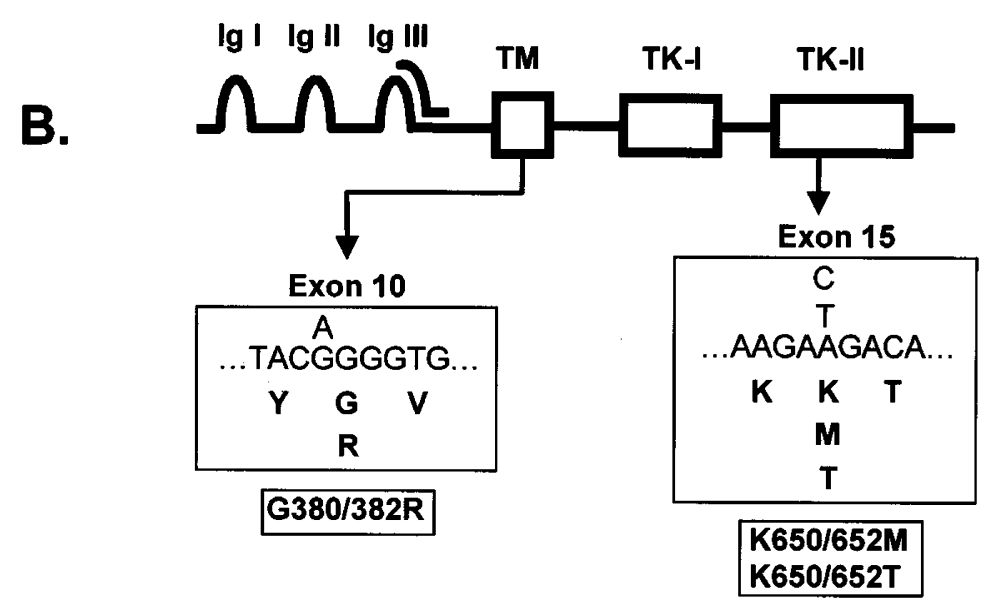

Figure 1 Identification of novel FGFR3 mutations in bladder cancer. (A) Novel somatic FGFR3 mutations were identified by PCR-SSCP followed by direct DNA sequencing. The tumour shown on the left side has a $G$ to $A$ transition in exon 10, which changes the sequence of codon 380/382 from GGG (Gly) to AGG (Arg). The tumour in the middle has an A to T transversion in exon 15, which changes the sequence of codon 650/652 from AAG (Lys) to ATG (Met). The tumour on the right side has an A to C transversion in exon 15, which alters the sequence of codon 650/652 from AAG (Lys) to ACG (Thr). Arrowheads indicate the positions of the mutations. The FGFR3b isoform, which is expressed in epithelial cells, contains two amino acids more than the FGFR3c isoform expressed in bone. Therefore, the G380R, K650M and K650T mutations in FGFR3 C are equivalent to the G382R, K652M and K652T mutations in FGFR3b. Note in addition: while the present study was under evaluation, the $\mathrm{K} 650 / 652 \mathrm{M}$ mutation in bladder cancer was reported by Kimura et al. ${ }^{26} \mathrm{~N}$, sequence of normal DNA; T, sequence of tumour DNA. (B) Schematic diagram of the FGFR3 protein. The areas within the two exons, in which the new missense mutations occurred, are shown in greater detail. The nucleotide substitutions are shown above, the amino acid substitutions are shown at the bottom of the Figure.

Table 1 The patient characteristics and the histophathological data corresponding to the novel and the concurrent FGFR3 mutations in bladder carcinomas. The cases with the numbers 143, 172, 287 and 265 contained two concurrent FGFR3 mutations in one tumor. The cases 287, 213, 17 and 55 presented with the novel mutations reported here for the first time

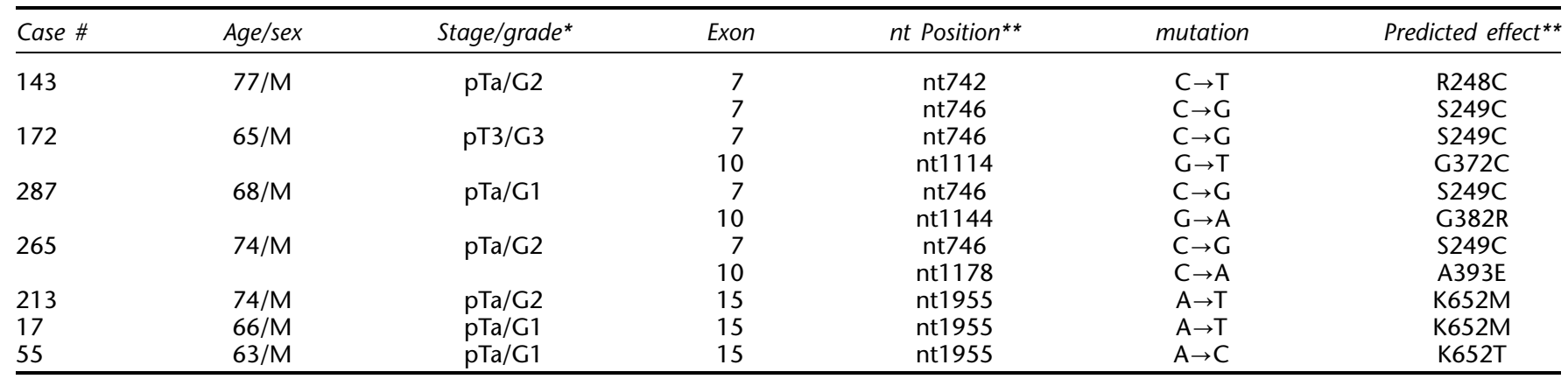

*Pathology according to the TNM97 and the WHO classification guidelines. ${ }^{* *}$ The nucleotide (nt) and codon numbering refer to the $F G F R 3 b$ isoform. 


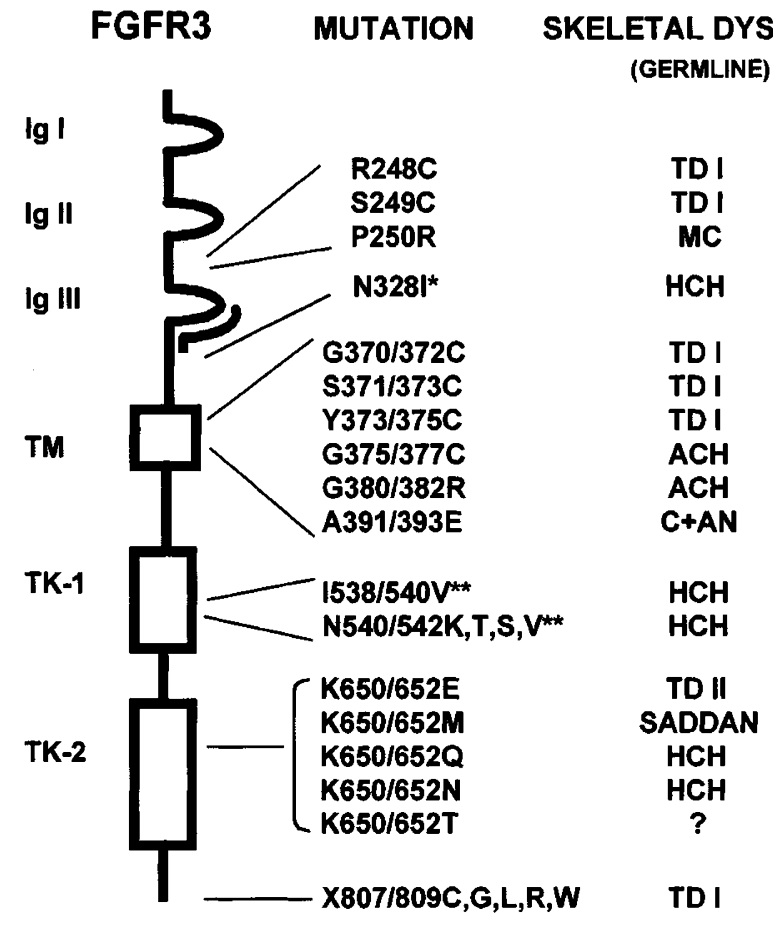

BLADDER CANCER (SOMATIC)

\author{
24 (8.1\%) \\ $214(71.8 \%)$ \\ $0(0 \%)$ \\ $0(0 \%)$ \\ $16(5.4 \%)$ \\ $0(0 \%)$ \\ $30(10.1 \%)$ \\ $0(0 \%)$ \\ $1(0.3 \%)$ \\ $5(1.7 \%)$ \\ $0(0 \%)$ \\ $0(0 \%)$ \\ $4(1.3 \%)$ \\ $2(0.7 \%)$ \\ $1(0.3 \%)$ \\ o $(0 \%)$ \\ $1(0.3 \%)$ \\ $0(0 \%)$
}

$n=298(100 \%)$

Figure 2 FGFR3 mutations associated with skeletal disorders and bladder carcinomas. A schematic diagram of the structure of FGFR3 is shown (IgG I-III, immunoglobulin-like domains; TM, transmembrane domain; TK-1 and -2, tyrosine kinase domains). The locations of the missense mutations associated with skeletal dysplasias (TDI, thanatophoric dysplasia type I; TDII, thanatophoric dysplasia type II; MC, Muenke craniosynostosis; $\mathrm{HCH}$, hypochondroplasia; $\mathrm{ACH}$, achondroplasia; $\mathrm{C}+\mathrm{AN}$, Crouzon syndrome with acanthosis nigricans; SADDAN, severe achondroplasia with developmental defect and acanthosis nigricans) and bladder carcinomas are indicated. The positions are numbered according to the FGFR $3 c / F G F R 3 b$ numbering. The number of occurrences and the percentage of each FGFR 3 mutation in mutated bladder tumours are given. These data were obtained from 570 bladder tumours of which 273 were reported before $5,16-18$ and 297 were reported here. The different mutations found in skeletal dysplasias have been obtained from the review of Passos-Bueno et al ${ }^{6}$ and the work of Bellus et $a l^{11}$ and Winterpacht et $a l^{27}{ }^{*}$ This region of FGFR3b corresponding to a region of FGFR3c where a mutation associated with hypochondroplasia (N328I) was previously found was only examined in 26 of the 570 bladder tumours studied so far. ${ }^{5}$ **This region containing mutations associated with hypochondroplasia was only examined in 89 of the 570 bladder tumours studied so far. $^{5,18}$

numbers are based on the previously published series by Cappellen et $a l^{5}$, Billerey et $a l^{16}$, van Rhijn et $a l^{17}$ and Sibley et $a l^{18}$ ( $n=273$ patients) and the series described in this paper ( $n=297$ patients).

\section{Discussion}

FGFR3 mutations occur frequently in bladder carcinoma. ${ }^{5,16-18}$ The vast majority of somatic FGFR3 mutations identified in bladder cancer are identical to those found in thanatophoric dysplasia. ${ }^{7,9}$ In the previous published series, only two FGFR3 mutations (A391/393E and K650/ $652 \mathrm{Q}$ ) did not correspond to thanatophoric dysplasia mutations. ${ }^{17,18}$ However, both mutations have been found to be associated with milder types of skeletal dysplasia: the A391/ 393E mutation with the Crouzon syndrome with acanthosis nigricans and the K650/652Q mutation with hypochondroplasia. In this new series of 297 bladder carcinomas reported here, we identified 181 FGFR3 mutations. Ninetysix per cent (173 out of 181) of these somatic mutations have been previously reported to be associated with thanatophoric dysplasia. However, other activating FGFR3 mutations including the three described here for the first time in bladder cancer (G380/382R, K650/652M and $\mathrm{K} 650 / 652 \mathrm{~T}$ ), have never been reported to be associated with thanatophoric dysplasia.

The activating G380/382R mutation, which affects the transmembrane domain, is responsible for almost all ( $\sim 97 \%)$ cases of achondroplasia. ${ }^{20,22}$ Achondroplasia is the most common form of non-lethal skeletal dysplasia, affecting approximately one in 15000 to one in 40000 live births. ${ }^{7}$ This mutation is here reported in cancer for the first time.

The activating $\mathrm{K} 650 / 652 \mathrm{M}$ mutation, located in the kinase domain, has already been found in SADDAN (severe 
achondroplasia with developmental delay and acanthosis nigricans) ${ }^{23}$ and in a primary multiple myeloma. ${ }^{12}$ Transient transfection studies have demonstrated that the K650/ $652 \mathrm{M}$ mutation results in stronger constitutive activation of FGFR3 than does the K650/652E mutation responsible for thanatophoric dysplasia. ${ }^{23}$ Nevertheless, despite multiple physical and neurological impairments, most SADDAN patients survive past infancy without the need for prolonged ventilator support. ${ }^{7,23}$

The K650/652T mutation has never been reported before in either skeletal dysplasia or cancer. However, other mutations of the same codon are associated with either thanatophoric dysplasia (K650/652E), SADDAN (K650/ $652 \mathrm{M}$ ) or hypochondroplasia (K650/652N and K650/ $652 \mathrm{Q}) .{ }^{10,11,23}$ Bellus et $a l^{11}$ investigated all the possible amino acid substitutions resulting from single nucleotide changes in the 650/652 codon and showed that the K650/ $652 \mathrm{~T}$ mutation leads to constitutive activation of the FGFR3 tyrosine kinase. This activation was equivalent to that observed with the K650/652N and K650/652Q (hypochondroplasia) mutations but was considerably weaker than observed with the K650/652E and K650/652M mutations associated with thanatophoric dysplasia and SADDAN, respectively.

The identification of mutations in bladder cancers identical $(\mathrm{K} 650 / 652 \mathrm{Q})^{18}$ or similar $(\mathrm{K} 650 / 652 \mathrm{~T})$ (this study) to mutations found in hypochondroplasia suggests that other hypochondroplasia mutations, such as the I538/540V and N540/542K,T,S,V mutations in exon 13 may also occur in this carcinoma. ${ }^{6,24}$ In other words, possible hypochondroplasia mutations in other exons than 15 may have escaped their detection in bladder cancer. Among the 570 bladder tumours studied so far, only 89 have been studied for the hypochondroplasia mutations located in exon 13. , $^{5}$ No activating mutations were detected in this exon for the 89 cases. Nevertheless, the percentage of mutations not corresponding to thanatophoric dysplasia in bladder cancer might be slightly higher than suggested.

Another novel finding presented here was the occurrence of two simultaneous FGFR3 mutations in four tumours (case-numbers: 143, 172, 265 and 287, Table 1). The PCR-SSCP analysis of exon 7 in the case with number 404 provided a strong indication that the two concurrent mutations (R248C and S249C), are carried by the two different alleles (not shown). For the remaining three cases, this remains to be determined because these tumours had no loss of heterozygosity at the FGFR3 locus (not shown) and RNA was not available.

From a clinical perspective, the presence of FGFR3 mutations in bladder carcinomas, identical to the mutations found in patients with non-lethal skeletal disorders (hypochondroplasia, achondroplasia, SADDAN and Crouzon syndrome) suggests that the patients with these FGFR3related syndromes might have a higher risk to develop bladder cancer than the general population. However, to our knowledge, there are no reports on a higher incidence of cancer in these patients, so it is possible that the non-lethal FGFR3 mutations do not sufficiently activate the protein to an extent that is needed for tumour formation or other genes must be affected for bladder tumour formation. On the other hand, the predisposition may have gone unnoticed to clinicians, especially if we consider the relative low prevalence of non-lethal skeletal syndromes $(\sim 10000$ cases in the USA). For example, the retinoblastoma gene, cloned 18 years ago, has been demonstrated to play a role in many carcinomas, including bladder carcinomas. Yet, it was only recently proven that hereditary retinoblastoma patients have a higher risk to develop lung cancer than unaffected individuals. ${ }^{25}$

\section{Acknowledgements}

This work was supported by the University Hospital Rotterdam as part of a top-down revolving fund project (FED 0930) and by grants from the Maurits and Anna de Kock foundations, the Comite de Paris Ligue Nationale Contre le Cancer (UMR 144, associated laboratory), the CNRS and the Institut Curie. The medical-ethical committee of the Erasmus University and the University Hospital Rotterdam approved the study (MEC 168.922/1998/55).

\section{References}

1 Johnson DE, Williams LT: Structural and functional diversity in the FGF receptor multigene family. Adv Cancer Res 1993; 60: 141.

2 Robertson SC, Tynan JA, Donoghue DJ: RTK mutations and human syndromes: when good receptors turn bad. Trends Genet 2000; 16: $265-271$.

3 Murgue M, Tsunekawa S, Rosenberg I, deBeaumont M, Podolsky DK: Identification of a novel variant of fibroblast growth factor receptor 3 (FGFR3 IIIb) in human colonic epithelium. Cancer Res 1994; 54: 5206-5211.

4 Delezoide AL, Benoist-Lasselin C, Legeai-Mallet L et al: Spatiotemporal expression of FGFR 1, 2 and 3 genes during human embryo-fetal ossification. Mech Dev 1998; 77: 19-30.

5 Cappellen D, De Oliveira C, Ricol D et al: Frequent activating mutations of FGFR3 in human bladder and cervix carcinomas. Nat Genet 1999; 23: 18-20.

6 Passos-Bueno MR, Wilcox WR, Jabs EW, Sertié AL, Alonso LG, Kitoh H: Clinical spectrum of fibroblast growth factor receptor mutations. Hum Mutat 1999; 14: 115-125.

7 Vajo Z, Francomano CA, Wilkin DJ: The molecular and genetic basis of fibroblast growth factor receptor 3 disorders: The achondroplasia family of skeletal dysplasias, Muenke craniosynostosis, and Crouzon syndrome with acanthosis nigricans. Endocr Rev 2000; 21: $23-39$.

8 Naski MC, Wang Q, Xu J, Ornitz DM: Graded activation of fibroblast growth receptor 3 by mutations causing achondroplasia and thanatophoric dysplasia. Nat Genet 1996; 13: 233-237.

9 Webster MK, Donoghue DJ: FGFR activation in skeletal disorders: too much of a good thing. Trends Genet 1997; 13: 178-182.

10 Tavormina PL, Shiang R, Thompson LM et al: Thanatophoric dysplasia (types I and II) caused by distinct mutations in fibroblast growth factor receptor 3. Nat Genet 1995; 9: 321-328.

11 Bellus GA, Spector EB, Speiser PW et al: Distinct missense mutations of the FGFR3 Lys650 codon modulate receptor kinase activation and the severity of the skeletal dysplasia phenotype. Am J Hum Genet 2000; 67: 1411-1421. 
12 Chesi M, Nardini E, Brents LA et al: Frequent translocation $\mathrm{t}(4 ; 14)(\mathrm{p} 16.3 ; \mathrm{q} 32.3)$ in multiple myeloma is associated with increased expression and activating mutations of fibroblast growth factor receptor 3. Nat Genet 1997; 16: 260-264.

13 Richelda R, Ronchetti D, Baldini L et al: A novel translocation $\mathrm{t}(4 ; 14)(\mathrm{p} 16.3 ; \mathrm{q} 32.3)$ in multiple myeloma involves the fibroblast growth-factor receptor 3 gene. Blood 1997; 90: 4062-4070.

14 Fracchiolla NS, Luminari S, Baldini L, Lombardi L, Maiolo AT, Neri A: FGFR3 gene mutations associated with human skeletal disorders occur rarely in multiple myeloma. Blood 1998; 92: 2987-2989.

$15 \mathrm{Wu}$ R, Connolly D, Ngelangel C, Bosch FX, Muñoz N, Cho KR: Somatic mutations of fibroblast growth factor receptor 3 (FGFR3) are uncommon in carcinomas of the uterine cervix. Oncogene 2000; 19: 5543-5546.

16 Billerey C, Chopin D, Aubriot-Lorton M-H et al: Frequent FGFR3 mutations in papillary non-invasive bladder (pTa) tumors. Am J Pathol 2001; 158: 1955-1959.

17 Van Rhijn BWG, Lurkin I, Radvanyi F, Kirkels WJ, Van der Kwast ThH, Zwarthoff EC: The fibroblast growth factor receptor 3 (FGFR3) mutation is a strong indicator of superficial bladder cancer with low recurrence rate. Cancer Res 2001; 61: 1265-1268.

18 Sibley K, Cuthbert-Heavens D, Knowles MA: Loss of heterozygosity at $4 \mathrm{p} 16.3$ and mutation of FGFR3 in transitional cell carcinoma. Oncogene 2001; 20: 686-691.

19 Meyers GA, Orlow SJ, Munro IR, Przylepa KA, Jabs EW: Fibroblast growth factor receptor 3 (FGFR3) transmembrane mutation in Crouzon syndrome with acanthosis nigricans. Nat Genet 1995; 11: $462-464$.
20 Rousseau F, Bonaventure J, Legeai-Mallet L et al: Mutations in the gene encoding fibroblast growth factor receptor-3 in achondroplasia. Nature 1994; 371: 252-254.

21 Shiang R, Thompson LM, Zhu YZ et al: Mutations in the transmembrane domain of FGFR3 cause the most common genetic form of dwarfism, achondroplasia. Cell 1994; 787: 335-342.

22 Bellus GA, Hefferon TW, Ortiz de Luna RI et al: Achondroplasia is defined by recurrent G380R mutations of FGFR3. Am J Hum Genet 1995; 56: $368-373$.

23 Tavormina PL, Bellus GA, Webster MK et al: A novel skeletal dysplasia with developmental delay and acanthosis nigricans is caused by a Lys650Met mutation in the fibroblast growth factor receptor 3 gene. Am J Hum Genet 1999; 64: 722 - 731.

24 Bellus GA, McIntosh I, Smith EA et al: A recurrent mutation in the tyrosine kinase domain of fibroblast growth factor receptor 3 causes hypochondroplasia. Nat Genet 1995; 10: 357-359.

25 Kleinerman RA, Tarone RE, Abramson DH, Seddon JM, Li FP, Tucker MA: Hereditary retinoblastoma and risk of lung cancer. $J$ Natl Cancer Inst 2000; 92: 2037-2039.

26 Kimura T, Suzuki H, Ohashi T, Asano K, Kiyota H, Eto Y: The incidence of thanatophoric dysplasia mutations in FGFR3 gene is higher in low-grade or superficial bladder carcinomas. Cancer 2001; 92: 2555-2561.

27 Winterpacht A, Hilbert K, Stelzer C et al: A novel mutation in FGFR-3 disrupts a putative N-glycosylation site and results in hypochondroplasia. Physiol Genomics 2000; 2: 9-12. 